\title{
Detection of Minimal Hepatic Encephalopathy in Iranian Patients with Cirrhosis
}

\author{
Mahsa Khodadoostan ${ }^{1}$, Sina Sadeghian ${ }^{2,}$, Ali Safaei ${ }^{2}$, Milad Kabiri ${ }^{3}$, \\ Sara Shavakhi ${ }^{4}$, Ali Reza Shavakhi ${ }^{4}$, Ahmad Shavakhi ${ }^{5}$
}

\begin{abstract}
1. Assistant Professor of Gastroenterology and Hepatology, Department of Gastroenterology and Hepatology, AlZahra Hospital, Isfahan University of Medical Sciences, Isfahan, Iran

2. Student of Medical Sciences, Department of Gastroenterology and Hepatology, Al-Zahra Hospital, Isfahan University of Medical Sciences, Isfahan, Iran

3. Student of Medical Sciences, Department of Gastroenterology and Hepatology, Mazandaran University of Medical Sciences, Mazandaran, Iran

4. Student of Medical Sciences, Department of Gastroenterology and Hepatology, Shahr-e-kord University of Medical Sciences, Shahr-e-kord, Iran

5. Professor of Gastroenterology and Hepatology, Department of Gastroenterology and Hepatology, Al-Zahra Hospital, Isfahan University of Medical Sciences, Isfahan, Iran
\end{abstract}

\section{* Corresponding Author:}

Sina Sadeghian, MD Department of Gastroenterology and Hepatology, Al-Zahra Hospital, Isfahan University of Medical Sciences, Isfahan, Iran

Telefax: + 983136412358

Email: sadeghian.sina72@gmail.com

Received: 09 May 2018

Accepted: 01 Sep. 2018

\section{ABSTRACT}

\section{BACKGROUND}

Minimal hepatic encephalopathy (MHE) is the mildest type of hepatic encephalopathy in patients with cirrhosis. Patients with MHE have normal clinical and physical examination but they show some neurocognitive dysfunctions that affect their quality of life negatively. The aim of the current study is to diagnose MHE in patients with cirrhosis and its associated factors.

\section{METHODS}

This is a cross-sectional study on 120 known cases of cirrhosis referred to hospitals affiliated to Isfahan University of Medical Sciences during 2014-17. The patients' cirrhosis severity was evaluated using laboratory tests and physical examinations based on MELD (Model for End-stage Liver Disease) and Child-Pugh criteria. The patients' demographics were filled in a checklist. All included patients with cirrhosis were asked to respond to the questions of Psychometric Hepatic Encephalopathy Score (PHES) test.

\section{RESULTS}

Mean age of the patients was $51.2 \pm 9.7$ years. 62 (51.7\%) patients were men and 58 (48.3\%) patients were women. The mean score of the patients based on MELD criteria was $14.03 \pm 6.09$. $26.7 \%$ of the patients presented MHE. Mean age of the patients with MHE was statistically less than the patients without MHE ( $p$ value $<0.001$ ). Mean score of MELD criteria among the patients with diagnosis of MHE was significantly higher than the other group ( $p$ value $<0.001$ ). The patients' Child class was statistically associated with $\operatorname{MHE}(p$ value $<0.001)$. Men were significantly more affected than women $(p$ value $=0.03)$.

\section{CONCLUSION}

MHE was associated with MELD score and Child class of the patients with cirrhosis. The noticeable point was reversible association of age with MHE. Further studies are recommended.

\section{KEYWORDS:}

Cirrhosis, Minimal hepatic encephalopathy, MELD score, Child-Pugh class

Please cite this paper as:

Khodadoostan M, Sadeghian S, Safaei A, Kabiri M, Shavakhi S, Shavakhi AR, Shavakhi A. Detection of Minimal Hepatic Encephalopathy in Iranian Patients with Cirrhosis. Middle East J Dig Dis 2018;10:230-235. doi: 10.15171/mejdd.2018.115.

\section{INTRODUCTION}

Hepatic encephalopathy (HE) is a common serious complication of cirrhosis, which is associated with spectrum of neuropsychological dysfunctions. HE can significantly cause morbidity and mortality in such patients. ${ }^{1}$

Various etiologies have been provided for HE, of them the most common one is the theory of endogen neurotoxic metabolites of ammonia produced by the body. These products should be metabolized in the liver while patients with cirrhosis have lost this ability. ${ }^{2}$ 
Table 1: Hepatic encephalopathy grading

\begin{tabular}{lc}
\hline $\begin{array}{l}\text { Hepatic encephalopathy } \\
\text { type }\end{array}$ & Manifestations \\
\hline I & $\begin{array}{c}\text { Changes in behavior, mild confusion, } \\
\text { slurred speech, disordered sleep }\end{array}$ \\
\hline II & Lethargy, moderate confusion \\
\hline III & $\begin{array}{c}\text { Marked confusion (stupor), incoherent } \\
\text { speech, sleeping but arousable }\end{array}$ \\
\hline IV & Coma, unresponsive to pain \\
\hline
\end{tabular}

$\mathrm{HE}$ is a condition that based on signs and symptoms has been divided to four subgroups (table 1). ${ }^{3} \mathrm{HE}$ may have some complications including pneumonia aspiration, cortical blindness, brain edema, and neurological deficits. The mildest form of HE is known as minimal hepatic encephalopathy (MHE). ${ }^{2}$ Patients with MHE have normal clinical and physical examination of mental status but such patients show some cognitive dysfunctions that can only be diagnosed through neuropsychological and neurophysiological tests. ${ }^{2,4}$ Patients with MHE may improve, remain unchanged, or deteriorate and develop overt $\mathrm{HE}$ over a long-term follow-up. The areas with impairments in patients with MHE are attention, visuospatial perception, speed of information processing, especially in the psychomotor area, fine motor skills, and short-term memory. 5,6

MHE shows mild cognitive function changes of electrophysiological parameters, neurotransmitter homeostasis, brain blood flow, and brain metabolism. Although these changes are not easily recognizable in cirrhotic patients with $\mathrm{MHE}^{7}$ but it can affect health related quality of life in these patients negatively. These effects include information processing speed reduction, personality disorders, sleep disorders, and motor dysfunction. One of the considerable points in such patients is their relative inability in performing personal tasks such as driving. ${ }^{8,9}$

The prevalence of MHE has been from $30 \%$ to $84 \%$ in patients with cirrhosis. These differences may be due to different means of measurements and also variety of societies. ${ }^{4,10}$

There is no evidence showing that the prevalence of MHE among Iranian patients with cirrhosis has been assessed previously. In addition, studies of other communities in this term, are limited and with small study population. Considering the importance of MHE diagnosis and few numbers of studies about this issue, we have decided to conduct the current study to find MHE in patients with cirrhosis.

\section{MATERIALS AND METHODS}

This is a cross-sectional study on 120 known cases of cirrhosis referred to Al-Zahra Hospital, a referral center for gastrointestinal diseases affiliated to Isfahan University of Medical Sciences during 2014-17.

Patients with the minimum age of 18 years and diagnosis of cirrhosis based on laboratory tests, radiological images, and/or biopsy sampling were included. Those with overt encephalopathy, known intracranial lesions, active gastrointestinal bleeding, progressive active infection, renal function impairment (serum creatinine $>2 \mathrm{mg} / \mathrm{dL}$ ), electrolyte disturbances (serum sodium $<130$ or $>150 \mathrm{meq} / \mathrm{dL}$ and/or serum potassium $<3.0$ or $>5.5 \mathrm{meq} / \mathrm{dL}$ ) and patients who were receiving treatment for $\mathrm{HE}$ such as lactulose or antibiotic for the previous 8 weeks or with the history of benzodiazepines, narcotics, opioids and/or alcohol use in this duration were excluded from the study. In addition, patients who were not able to answer the questions for any reason were excluded as well. Also, all patients with signs and symptoms of moderate or severe HE including asterixis, confusion, lethargy, seizure, and personality and thinking changes in their history and physical examination were excluded from the study.

The method of sampling was non-randomized consecutive. In this order, all patients who were referred to Al-Zahra Hospital were assessed until the study population was completed.

The severity of the patients' cirrhosis was evaluated using laboratory tests and physical examinations based on MELD (Model for End-stage Liver Disease) and Child-Pugh criteria. ${ }^{11}$

All included patients were interviewed by a single gastrointestinal specialist and were asked to respond to the questions of Psychometric Hepatic Encephalopathy Score (PHES) test. ${ }^{2,12}$ PHES includes number connection tests $\mathrm{A}$ and $\mathrm{B}$, the digit symbol test, the line tracing test, and the circle dotting test. ${ }^{5}$

The score was measured for each patient according to their age and level of education (PHES RedeH). ${ }^{13}$ In case of score $<4$ or complete inability to perform two numbers of the above tests, the patient was diagnosed as having MHE.

The data were analyzed using IBM SPSS software version 20. Descriptive data were reported in mean \pm standard deviation. For analytic data, Chi-square and ANOVA tests were used. $P$ values less than 0.05 were considered as statistically significant. 
Table 2: Distribution of etiology, Child-Pugh class, and presence of minimal hepatic encephalopathy in patients

\begin{tabular}{|c|c|c|c|}
\hline Variables & & $\mathbf{N}$ & $\%$ \\
\hline \multirow[t]{5}{*}{ Etiology } & Viral & 51 & 42.5 \\
\hline & Non-alcoholic hepatic failure & 19 & 15.8 \\
\hline & Alcoholic hepatic failure & 20 & 16.7 \\
\hline & Not known & 9 & 7.5 \\
\hline & Others & 21 & 17.5 \\
\hline \multirow[t]{3}{*}{ Child-Pugh class } & A & 78 & 65 \\
\hline & B & 37 & 30.8 \\
\hline & $\mathrm{C}$ & 5 & 4.2 \\
\hline \multirow{2}{*}{$\begin{array}{l}\text { Presence of minimal hepatic } \\
\text { encephalopathy }\end{array}$} & Yes & 32 & 26.7 \\
\hline & No & 88 & 73.3 \\
\hline
\end{tabular}

Table 3: Mean age and cirrhosis severity based on MELD considering MHE status (Model for End-stage Liver Disease; Minimal hepatic encephalopathy)

\begin{tabular}{lccc}
\hline Variable & MHE positive $($ mean \pm SD) & MHE negative (mean \pm SD) & \multicolumn{1}{c}{ value } \\
\hline Age & $44.3 \pm 9.1$ & $53.8 \pm 8.7$ & $<0.00$ \\
\hline MELD score & $21.8 \pm 5.8$ & $11.2 \pm 2.8$ & $<0.001$ \\
\hline
\end{tabular}

Table 4: MHE distribution based on Child-Pugh class, sex, and etiology (MHE: Minimal hepatic encephalopathy)

\begin{tabular}{|c|c|c|c|}
\hline Variables & & MHE (number (\%)) & $P$ value \\
\hline \multirow[t]{3}{*}{ Child-Pugh class } & A & $3(3.8 \%)$ & \multirow{3}{*}{$<0.001$} \\
\hline & B & $24(64.9 \%)$ & \\
\hline & $\mathrm{C}$ & $5(100 \%)$ & \\
\hline \multirow[t]{2}{*}{ Sex } & Female & $11(19 \%)$ & \multirow{2}{*}{0.03} \\
\hline & Male & $21(33.9 \%)$ & \\
\hline \multirow[t]{5}{*}{ Etiology } & Viral & $13(25.5 \%)$ & \multirow{5}{*}{0.74} \\
\hline & Non-alcoholic hepatic failure & $4(21 \%)$ & \\
\hline & Alcoholic hepatic failure & $6(30 \%)$ & \\
\hline & Not known & $4(44.4 \%)$ & \\
\hline & Others & $5(23.8 \%)$ & \\
\hline
\end{tabular}

\section{RESULTS}

In the current study, 120 patients with cirrhosis with the mean age of $51.2 \pm 9.7$ years (range: $27-75$ years) were evaluated. 62 patients $(51.7 \%)$ were men and 58 (48.3\%) were women.

The mean MELD score of the patients was $14.03 \pm 6.09$. Information about cirrhosis status of the patients based on Child-Pugh criteria and presence of MHE is demonstrated in table 2.

MHE was diagnosed in 32 patients (26.7\%). Mean age of the patients affected with MHE was less than cirrhotic patients without MHE $(P<0.001)$. Mean score of MELD criteria among the patients with diagnosis of MHE was significantly higher than the other group $(P<0.001)$ (table 3$)$.
Rate of MHE was significantly higher among men $(P=0.03)$ but it was not in association with the etiology of cirrhosis $(P=0.74)$.

There was a significant association between ChildPugh class and MHE. In this term, the patients with class $\mathrm{C}$ cirrhosis were affected more than the patients class $\mathrm{B}$ and those with class $\mathrm{B}$ were affected more than the patients with class A (table 4).

\section{DISCUSSION}

MHE is a crucial reversible condition that affects patients' quality of life and daily activity steeply and negatively. Several studies have recommended MHE therapy due to its improvement (Several studies have insisted on 
reversibility of hepatic encephalopathy in its minimal phase; thus therapeutic considerations for prevention and treatment of this condition is recommended). In fact treatment of MHE can delay its progression and also improve the quality of life thus promotes life expectancy. On the other hand, based on time-consuming neuropsychological tests, hepatologists rarely spend time to screen patients with MHE. ${ }^{14-16}$ What has been mentioned above shows the importance of diagnosing MHE in patients with cirrhosis.

Different studies have presented various prevalence of MHE among patients with cirrhosis. These differences may have occurred based on the type of neurophysiological and neuropsychological screening tests that they have used for their patients. Generally, the prevalence of MHE has been estimated from $20 \%$ to $80 \%$ in various studies. ${ }^{4,17}$ In the current study we detected MHE in $26.7 \%$ of our patients while Praveen Sharma and colleagues in another study conducted in the Middle East reported MHE in $41 \%$ of their assessed patients. ${ }^{18}$

We found that MELD score was significantly associated with the incidence of MHE. In addition, Child class as a manifestation of hepatic failure severity and also a predictor of surveillance of patients with cirrhosis has been found in accordance with the presence of MHE. In fact, we found that $100 \%$ of the patients with Child class $\mathrm{C}$ presented $\mathrm{HE}$ while $64.9 \%$ of the patients with Child class B and $3.8 \%$ of the patients with Child class A presented MHE. These results are comparable with other studies as previous reports have presented controversial results. For instance, in a study by Praveen Sharma and others MHE was significantly associated with MELD score and MELD score $>15.5$ was in accordance with the incidence of MHE. Similarly, Meyer and co-workers found that the higher MELD score the more trail-making test A and B impaired. ${ }^{19}$ This result is in contrast to the study of Yoo and colleagues who found no association between the patients' MELD score and neurophysiological study using electroencephalogram. ${ }^{20}$ The next point is assessment of Child class with the neurocognitive function of patients with cirrhosis. Chen and co-workers in their study presented that Child score was independently associated with MHE and also patients' surveillance. ${ }^{21}$

Age and sex distribution of MHE among patients with cirrhosis was the next assessment of our study. Although sex distribution of the studied patients was not notably different but MHE occurrence was significantly higher among men. This sex distribution may show the severity of cirrhosis, which may be worse among men as we have not compared Child class between men and women. It should be mentioned that there are studies that have presented results similar to ours such as the studies by Chen and Goyal and their co-workers that reported male involvement in over $70 \%$ of their patients. ${ }^{21,22}$

Another considerable point about our patients is the age of the patients at the time of MHE detection. In the current study the patients with MHE were significantly younger than those without hepatic encephalopathy. It can be hypothesized that younger patients were more sensitive to level of ammonia in comparison with elder patients. Also other hypothesis can be considered such as earlier onset in younger cases, or maybe higher ChildPugh score of younger patients, which we have not assessed in the current study. Further studies are recommended. Chen and colleagues presented diverse results as they found no association between MHE affected patients' age and not-affected ones. ${ }^{21} \mathrm{Yu}$-Yuan Li and co-workers found higher rate of MHE in their older patients but they have presented that MHE occurrence was in relation with the patients' level of education as well. ${ }^{23}$ The results of the study by Mina and colleagues were somewhat different as they found MHE in their older patients but not significantly. ${ }^{4}$

\section{CONCLUSION}

Based on our findings, MHE was associated with the MELD score and Child class of the patients with cirrhosis. In addition men were more affected by MHE. The noticeable point about our patients was the association between age and the incidence of MHE as we found a reverse association between the patients' age and MHE incidence. Further studies in this matter are recommended.

\section{Limitations:}

In the current study we have not assessed patients' age at the onset of cirrhosis, duration of cirrhosis, and the level of ammonia.

\section{Financial Support:}

The study was sponsored by Isfahan University of Medical Sciences. 


\section{ACKNOWLEDGEMENTS}

We are grateful to Alzahra Hospital's head office of Gastroenterology ward, nurses, and employees.

\section{ETHICAL APPROVAL}

There is nothing to be declared.

\section{CONFLICT OF INTEREST}

The authors declare no conflict of interest related to this work.

\section{REFERENCES}

1. Ferenci P, Lockwood A, Mullen K, Tarter R, Weissenborn K, Blei AT. Hepatic encephalopathy-definition, nomenclature, diagnosis, and quantification: final report of the working party at the $11^{\text {th }}$ World Congresses of Gastroenterology, Vienna, 1998. Hepatology 2002;35:716-21. doi:10.1053/jhep.2002.31250.

2. Benedeto-Stojanov D, Stojanov D. Minimal Hepatic Encephalopathy (MHE). Miscellanea on EncephalopathiesA Second Look: InTech; 2012.

3. Vilstrup H, Amodio P, Bajaj J, Cordoba J, Ferenci P, Mullen $\mathrm{KD}$, et al. Hepatic encephalopathy in chronic liver disease: 2014 Practice Guideline by the American Association for the Study of Liver Diseases and the European Association for the Study of the Liver. Hepatology 2014;60:715-35. doi:10.1002/hep.27210.

4. Mina A, Moran S, Ortiz-Olvera N, Mera R, Uribe M. Prevalence of minimal hepatic encephalopathy and quality of life in patients with decompensated cirrhosis. Hepatol Res 2014;44:E92-9. doi:10.1111/hepr.12227.

5. Barbosa M, Marinho C, Mota P, Cotter J. Minimal Hepatic Encephalopathy: The Reality Beyond Our Eyes. Acta Med Port 2015;28:480-5.

6. Zhan T, Stremmel W. The diagnosis and treatment of minimal hepatic encephalopathy. Dtsch Arztebl Int 2012;109:180-7. doi:10.3238/arztebl.2012.0180.

7. Moriwaki H, Shiraki M, Iwasa J, Terakura Y. Hepatic encephalopathy as a complication of liver cirrhosis: anAsian perspective. J Gastroenterol Hepatol 2010;25:858-63. doi:10.1111/j.1440-1746.2010.06242.x.

8. Stewart CA, Malinchoc M, Kim W, Kamath PS. Hepatic encephalopathy as a predictor of survival in patients with end-stage liver disease. Liver Transpl 2007;13:1366-71. doi:10.1002/1t.21129.

9. Rivera Ramos JF, Rodríguez Leal C. Review of the final report of the 1998 Working Party on definition, nomenclature and diagnosis of hepatic encephalopathy. Ann Hepatol 2011;10(Suppl 2):S36-S9.
10. Wunsch E, Szymanik B, Post M, Marlicz W, Mydłowska M, Milkiewicz P. Minimal hepatic encephalopathy does not impair health-related quality of life in patients with cirrhosis: a prospective study. Liver International 2011;31:980-4. doi:10.1111/j.1478-3231.2011.02465.x.

11. Angermayr B, Cejna M, Karnel F, Gschwantler M, Koenig F, Pidlich J, et al. Child-Pugh versus MELD score in predicting survival in patients undergoing transjugular intrahepatic portosystemic shunt. Gut 2003;52:879-85. doi:10.1136/gut.52.6.879.

12. Duarte-Rojo A, Estradas J, Hernández-Ramos R, Ponce-deLeón S, Córdoba J, Torre A. Validation of the psychometric hepatic encephalopathy score (PHES) for identifying patients with minimal hepatic encephalopathy. Dig Dis Sci 2011;56:3014-23. doi:10.1007/s10620-011-1684-0.

13. Badea MA, Drug VL, Dranga M, Gavrilescu O, Stefanescu G, Popa I, et al. Diagnosis of minimal hepatic encephalopathy in a tertiary care center from eastern Romania: validation of the psychometric hepatic encephalopathy score (PHES). Metab Brain Dis 2016;31:1463-71. doi:10.1007/ s11011-016-9878-y.

14. Bajaj JS, Etemadian A, Hafeezullah M, Saeian K. Testing for minimal hepatic encephalopathy in the United States: an AASLD survey. Hepatology 2007;45:833-4. doi:10.1002/hep.21515.

15. Prasad S, Dhiman RK, Duseja A, Chawla YK, Sharma A, Agarwal R. Lactulose improves cognitive functions and health-related quality of life in patients with cirrhosis who have minimal hepatic encephalopathy. Hepatology 2007;45:549-59. doi:10.1002/hep.21533.

16. Weissenborn K, Giewekemeyer K, Heidenreich S, Bokemeyer M, Berding G, Ahl B. Attention, memory, and cognitive function in hepatic encephalopathy. Metab Brain Dis 2005;20:359-67. doi:10.1007/s11011-005-7919-z.

17. Vilstrup H, Amodio P, Bajaj J, Cordoba J, Ferenci P, Mullen $\mathrm{KD}$, et al. Hepatic encephalopathy in chronic liver disease: 2014 Practice Guideline by the American Association for the Study of Liver Diseases and the European Association for the Study of the Liver. Hepatology 2014;60:715-35. doi:10.1002/hep.27210.

18. Sharma P, Sharma BC. Predictors of minimal hepatic encephalopathy in patients with cirrhosis. Saudi J Gastroenterol 2010;16:181-7. doi:10.4103/1319-3767.65189.

19. Meyer T, Eshelman A, Abouljoud M. Neuropsychological changes in a large sample of liver transplant candidates. Transplant Proc 2006;38:3559-60. doi:10.1016/j. transproceed.2006.10.050

20. Yoo HY, Edwin D, Thuluvath PJ. Relationship of the model for end-stage liver disease (MELD) scale to hepatic encephalopathy, as defined by electroencephalography and neuropsychometric testing, and ascites. Am J Gastroenterol 2003;98:1395-9. doi:10.1111/j.1572-0241.2003.07466.x. 
21. Chen HJ, Chen R, Yang M, Teng GJ, Herskovits E. Identification of minimal hepatic encephalopathy in patients with cirrhosis based on white matter imaging and Bayesian data mining. AJNR Am J Neuroradiol 2015;36:481-7. doi:10.3174/ajnr.A4146.

22. Goyal O, Sidhu SS, Kishore H. Minimal hepatic encephalopathy in cirrhosis-how long to treat? Ann Hepatol 2017;16:115-22. doi:10.5604/16652681.1226822.

23. Li YY, Nie YQ, Sha WH, Zeng Z, Yang FY, Ping L, et al. Prevalence of subclinical hepatic encephalopathy in cirrhotic patients in China. World $J$ Gastroenterol 2004;10:2397-401. doi:10.3748/wjg.v10.i16.2397. 